\title{
Multi-Organ System Dysfunction in Covid-19 - A Review
}

\author{
Pooja Bhadoria ${ }^{1}$, Harsha Rathore ${ }^{2}$ \\ 1, 2 Department of Anatomy, All India Institute of Medical Sciences, Rishikesh, Uttarakhand, India.
}

\section{ABSTRACT}

With a continuous increase in Covid-19 cases, patients infected with SARS-CoV-2 have shown a wide variety of symptoms including fever, shortness of breath, cough, myalgia, dizziness, headache, impaired consciousness, abdominal pain and diarrhoea. Lab studies have shown higher leukocyte counts and neutrophil-lymphocyte ratio (NLR), decreased lymphocytes, increased prothrombin time and a partial thromboplastin time, increased serum lactate dehydrogenase, ferritin and various other markers, indicating multiple organ system dysfunction including respiratory system, immune system, nervous system and gastrointestinal tract. Apart from these, heart and kidneys are also found to be affected in some patients. Patients presenting with previous history of diseases such as cardiovascular diseases, kidney diseases, pregnant females, neonates, immunosuppressed patients, and aged people have shown poor prognosis of disease. Various studies suggest possible reasons of multiple organ dysfunctions including wide distribution of angiotensin-converting enzyme 2 (ACE-2) receptors, cytokine storm and hypercoagulability of blood. We have listed the various organ systems affected by the virus and possible explanations as to why and how these organ systems are affected.

SARS-CoV-2 outbreak has caused a global pandemic, with more than $33,400,000$ confirmed cases of Covid-19, including more than 1,000,000 deaths, reported to World Health Organization (WHO) globally till 30 September $2020 .{ }^{1}$ with a basic reproductive number estimated to be $2.2(1.4-3.9)$ the virus spread is very fast affecting many individuals across the globe. ${ }^{2}$

One of the major causes of efficient spread of SARS-CoV-2 in humans is the angiotensin-converting enzyme 2 receptors, used by the virus to enter the cells. ${ }^{3}$ Hence, wide distribution of angiotensin-converting enzyme 2 receptors in body including lungs, kidney, bladder, gastrointestinal tract, heart, central nervous system and peripheral nervous system ${ }^{4}$ and even placenta ${ }^{5}$ is one of the reasons the virus is found to affect these organs.

Two closely related viruses causing Middle East respiratory syndrome (MERS) and severe acute respiratory syndrome (SARS) were also found to affect multiple organ systems. SARS-CoV-1 was found in the respiratory pathway (trachea, bronchus, lung), gastrointestinal tract (stomach, small intestines), glands (sweat gland, liver, pancreas, parathyroid, pituitary, adrenal gland), renal system (convoluted renal tubules) and brain tissue (cerebrum) in the autopsy samples of patients who died because of severe acute respiratory syndrome. ${ }^{6}$ Respiratory, circulatory, gastrointestinal, haematological, renal and hepatological symptoms were also found to be affected in patients with Middle East respiratory syndrome infection.7 Though the virus is found to affect multiple organ systems, the underlying mechanisms are not clearly understood.

\author{
Corresponding Author: \\ Dr. Pooja Bhadoria, \\ Level-2, Block $A$, \\ Department of Anatomy, \\ All India Institute of Medical \\ Sciences, Rishikesh, \\ Uttarakhand, India. \\ E-mail: pooja.ana@aiimsrishikesh.edu.in
}

DOI: $10.14260 / j e m d s / 2021 / 135$

How to Cite This Article: Bhadoria P, Rathore H. Multi-organ system dysfunction in Covid-19 - a review. J Evolution Med Dent Sci 2021;10(09):632637, DOI: 10.14260/jemds/2021/135

Submission 21-10-2020,

Peer Review 02-01-2021,

Acceptance 09-01-2021,

Published 01-03-2021.

Copyright (C) 2021 Pooja Bhadoria et al. This is an open access article distributed under Creative Commons Attribution License [Attribution 4.0 International (CC $B Y 4.0)]$ 


\section{IMMUNE SYSTEM}

With infection of an individual with pathogens, immune system is activated. Lymphocytopenia is often seen in Covid19 patients with increased total white blood count. High neutrophil-lymphocyte ratio and lower percentages of eosinophils, monocytes and basophils ${ }^{7}$ are commonly seen in Covid-19 patients. A very low eosinophil count may indicate a poor prognosis of disease. ${ }^{8}$ The number of $\mathrm{T}$ cells were found to be significantly decreased. Most severe cases demonstrated elevated levels of infection-related biomarkers and inflammatory cytokines. ${ }^{7}$ The exaggerated immune response in patients with Covid-19 can cause the cytokine storm responsible for the involvement of multiple organ systems in disease.

\section{CYTOKINE STORM}

Cytokine storm is one of the mechanisms responsible for deterioration of multiple organ systems in Covid-19. Inflammation begins at tissue level and spreads via the circulation to other body parts. ${ }^{9}$ The damaged tissue releases inflammatory markers which activates the antigen presenting cells which further activates the immune system. Hence various antibodies and cytokines are released which leads to further local as well as systemic inflammation. Cytokines associated with the cytokine storm include interferons, interleukins, colony stimulating factors (CSFs), chemokines and tumour necrosis factors (TNFs). ${ }^{9}$ Inflammation and organ dysfunction are linked in a vicious cycle by a positive feedback mechanism. ${ }^{10}$

Severe lung infections as in the case of Covid-19 is, local inflammation can produce systemic sepsis by circulating inflammatory mediators. ${ }^{9}$ A characteristic plasma cytokine profiles changing with time is seen in studies of patients with severe sepsis because of pulmonary or extra pulmonary pathologies. The cytokines such as tumour necrosis factors and IL-1 $\beta$ and the chemotactic cytokines IL-8 and monocyte chemoattractant protein-1 (MCP-1) appear early in infection, followed by a more sustained increase in IL-6. Later the antiinflammatory cytokines (IL-10) also increases, as the body tries to control the over exaggerated inflammatory responses. ${ }^{9}$ An increased ferritin level is an indicative for cytokine storm. ${ }^{10}$ IL-2R and IL-6 serum levels may predict pneumonia severity as in Covid-19 and the prognosis of disease. ${ }^{11}$

\section{RESPIRATORY SYSTEM}

Respiratory failure is major cause of death among the patients with severe acute respiratory syndrome coronavirus-2 infection. ${ }^{12}$ Involvement of respiratory system is because of its direct connection with environment and high density of angiotensin-converting enzyme 2 receptors. The commonest symptoms of patients with SARS-CoV-2 are fever, fatigue and dry cough. ${ }^{13}$ Patients infected with severe acute respiratory syndrome also presented with similar symptoms of fever, shortness of breath, cough and myalgia. ${ }^{14}$ In another study among patients with Covid-19; most commonly observed complication was found to be sepsis followed by respiratory failure, acute respiratory distress syndrome (ARDS), heart failure and septic shock. ${ }^{15}$

\section{ARDS}

Pathophysiology of acute respiratory distress syndrome includes increased permeability of pulmonary capillaries because of capillary destruction and vasodilation secondary to entry of virus and inflammation. ${ }^{16}$ Because of vasodilation neutrophils accumulate in lungs and release cytotoxic molecules which cause tissue necrosis and induce autophagy and apoptosis of cells and result into further tissue damage. ${ }^{16,17}$ The damaged tissue releases various inflammatory markers which than induce the immune system and may lead to cytokine storm which further deteriorates the condition. Pro-inflammatory cytokines including TNF $\alpha$, IL-1 $\beta$, IL-8, IL-18 and IL-33 play major role in development of acute respiratory distress syndrome and may be used as markers of acute respiratory distress syndrome. Due to increased permeability and alveolar capillaries damage plasma contents accumulate in the alveolar space leading to development of pulmonary oedema and disturbing the pulmonary surfactant system, which leads to Respiratory Distress Syndrome (RDS) as in the cases of Covid-19.16

Continuous prone position ventilation (PRONE) is found to improve oxygenation in patients with severe acute respiratory distress syndrome. This technique is found to influence IL-6 expression in individuals with Severe Community Acquired Pneumonia (Chan et al., 2007). ${ }^{18}$

\section{CARDIOVASCULAR DISEASES IN COVID-19}

Covid-19 and cardiovascular diseases are found to have a twoway relationship. Covid-19 can aggravate damage to heart, on the other hand, patients already having a cardiovascular disease show poor prognosis. Patients of both severe acute respiratory syndrome and Middle East respiratory syndrome had shown cardiovascular manifestations. A study including 637 severe MERS CoV cases have shown the prevalence of cardiac diseases in $30 \%$, obesity in $16 \%$, diabetes in $50 \%$ and hypertension in $50 \%$ of total cases. ${ }^{19}$ In another study on Covid-19 in Wuhan the most frequently observed comorbidities were diabetes and hypertension, followed by cardiovascular disease. ${ }^{20}$ Inciardi et al. described a patient with Covid-19, left ventricular dysfunction and acute myopericarditis, without any previous history of cardiovascular diseases. ${ }^{21}$ Findings suggested that heart and cardiovascular system may involve in Covid-19 even without any noticeable respiratory dysfunction and other common symptoms of disease. Patients with Covid-19 infections are shown to develop various cardiovascular complications, such as vasculitis, heart failure, myocardial injury, arrhythmias and pericarditis. ${ }^{13,21}$ SARS-CoV-2 can cause the inflammation of heart muscles since it invades the cells using angiotensinconverting enzyme 2 receptors, though specific mechanisms are uncertain $^{22}$ The angiotensin-converting enzyme 2 receptors are extensively expressed in the cardiovascular system. About $7.5 \%$ cells of myocardium are shown to have 
angiotensin-converting enzyme 2 expression ${ }^{(4)}$. Abundance of angiotensin-converting enzyme 2 in heart muscle might be one of the reasons of cardiac involvement in patients with Covid19. Another proposed mechanism of myocardial injury is cytokine storm. Inflammatory cytokines like TNF, IL-1 and IL6 can be arrhythmogenic as they can affect the ion channel functions in cardiac musculature. ${ }^{23,24}$ These cytokines can favour the development of Long QT Segments by decreasing specific cardiac $\mathrm{K}+$ currents and increasing L-type Ca2+ currents $^{24}$ further contributing to arrhythmias. Other possible mechanisms of serious heart injury include hypoxemia and respiratory dysfunction seen in Covid-19 patients, resulting in damage to heart muscles. ${ }^{22}$

\section{HAEMATOLOGICAL}

Covid-19 patients are seen to be presented with various haematological findings including prolonged prothrombin time and partial thromboplastin time as markers of coagulation abnormalities; lactate dehydrogenase , C-reactive protein CRP, IL- 6 as inflammatory markers; increased ferritin, procalcitonin and D-dimer levels are associated with poor prognosis of disease..$^{25} \mathrm{~A}$ post mortem study of 12 patients died of Covid-19 revealed deep vein thrombosis (DVT) in $58 \%$ and pulmonary embolism was found to be the cause of death in 4 of them. ${ }^{26}$

Various thrombotic and ischemic haemorrhagic and coagulation related manifestations are present in patients with Covid-19. Though mechanisms involved in development of thrombosis in Covid-19 patients remain unclear; profound hypoxemia during endothelial inflammation may leads to vasoconstriction promoting vascular occlusion. ${ }^{27}$ Apart from this disturbed coagulation and fibrinolytic pathways in Covid19 leads to accumulation of fibrin in alveolar space which blocks normal gaseous exchange. ${ }^{27}$ Lupus anticoagulant which may contribute to thrombosis is found to be elevated in these patients apart from this D-dimer is also significantly elevated in these patients. ${ }^{27}$ Potential downregulation of angiotensinconverting enzyme 2 receptor expression can lead to increase tissue factor production and increased plasminogen activator inhibitor 1 production; also this down regulation of receptors along with increase in proinflammatory cytokines increase vascular injury and platelet action which may lead to hypercoagulable state leading to thrombosis in severe cases. ${ }^{28}$

\section{NERVOUS SYSTEM}

Both SARS-CoV and MERS-CoV are shown to possess neuroinvasive properties and have various neurological manifestations. In a study by Jiang et al., severe acute respiratory syndrome virus was detected in all the brain autopsies of patients died of severe acute respiratory syndrome. ${ }^{29}$ Various studies have suggested a similar property in SARS- CoV-2. Neurologic manifestations of Covid-19 include central nervous system manifestations such as headache, dizziness and impaired consciousness, acute cerebrovascular disease, peripheral nervous system manifestations including taste impairment, smell impairment and skeletal muscular injury manifestations. ${ }^{30}$ A patient with Covid-19 exhibiting loss of the involuntary process of breathing shows the involvement of brainstem in disease. ${ }^{30}$ Various neurological diseases are said to be associated with Covid-19. Several cases suggest a possible association between Guillain-Barré syndrome and SARS-CoV-2 infection. ${ }^{31}$ The virus is found to be associated with cases of encephalopathy ${ }^{32}$ and encephalitis. ${ }^{33}$

It is shown that coronaviruses can enter the central nervous system through at least 3 routes, via cranial nerve- 1 , by circulation, or by lymphatic system. ${ }^{34}$ Since angiotensinconverting enzyme 2 receptors are widespread in brain, it might be another explanation for nervous system involvement in Covid-19.

\section{OLFACTORY ROUTE}

A study of mouse hepatitis virus strain JHM (MHV-JHM) by Stenly et al. demonstrated a possible route for neurotropic virus to reach brain. Their studies suggest that after intranasal injection of virus, viral RNA might enter the mouse CNS via the cranial nerves innervating the nose i.e. Trigeminal and olfactory nerves. ${ }^{34}$ RNA traces were identified in various structures of olfactory tract including olfactory bulb, anterior olfactory nucleus, olfactory tubercle, the pyriform cortex, the diagonal band of Broca and Amygdala. ${ }^{34}$ RNA was also detected in trigeminal nerve tract including mesencephalic nucleus of trigeminal tract. This is a possible route by which the Covid-19 can also reach the brain.

\section{TRANSSYNAPTIC EXCHANGES OF CORONAVIRUSES}

In a study by Yan Chao Li et al. on Hemagglutinating Encephalomyelitis Virus (HEV67N) coronavirus in Rat central nervous system, virus progeny was found to enter the next order neuron by endocytosis which provides an evidence for transsynaptic exchange of coronaviruses. ${ }^{35}$

\section{RENAL INVOLVEMENT}

In a study at Wuhan of a total of 701 patients, approximately $13 \%$ of patients had underlying kidney disease. More than 40 $\%$ had abnormal kidney functions and $5.1 \%$ had acute kidney injury (AKI) during their stay at hospital. Patients presenting with kidney disease on admission and acute kidney injury during hospitalization were associated with an increased risk of mortality. Risk of death increases with increasing stages of acute kidney injury. ${ }^{36}$ The involvement of kidney is also shown by the severe acute respiratory syndrome virus with development of acute renal impairment in $6.7 \%$ of patients. 37 Underlying causes of kidney damage in patients infected with Covid-19 may include the presence of high density of angiotensin-converting enzyme 2 receptors in kidney, hypoxia, cytokine storm and thrombosis.

angiotensin-converting enzyme 2 receptors are detected in renal tubules and glomeruli. angiotensin-converting enzyme 2 receptors are also expressed in endothelium of interlobular arteries as well as vascular smooth muscle cells. While neo-expression of angiotensin-converting enzyme 2 
was found in glomerular and peritubular capillary endothelium in cases of primary and secondary renal diseases as well as renal transplants. ${ }^{38}$ This increased neo-expression of angiotensin-converting enzyme 2 may explain the poor prognosis of patients already having kidney diseases. Angiotensin-converting enzyme 2 receptors are found to express more in kidneys as compared to lungs. ${ }^{39}$

Renal histopathological analysis of 26 post-mortem findings of patients with Covid-19 in China, revealed diffuse proximal tubule injury with the loss of brush border, nonisometric vacuolar degeneration and frank necrosis. Identification of occasional hemosiderin granules and pigmented casts were also identified. Red blood cell clusters were detected which obstructs the capillary lumen may lead to kidney damage by hypoxia. Electron microscopic studies showed the presence of clusters of coronavirus and angiotensin-converting enzyme 2 receptors were also found to be increased. In addition to the direct effects of SARS-CoV-2 because of high density of angiotensin-converting enzyme 2 in kidney, other factors contributing to acute kidney injury may include hypoxia, coagulation abnormalities and possible drug or hyperventilation-rhabdomyolysis. ${ }^{40}$

\section{GIST}

Gastrointestinal tract is one of the organ systems having highest density of angiotensin-converting enzyme 2 receptors. Very high angiotensin-converting enzyme 2 density was found in gastrointestinal tract with approximately $30 \%$ of ileal epithelial cells and more than $1 \%$ oesophagus epithelial cells being angiotensin-converting enzyme 2 positive. The cells from stomach and liver showed lower angiotensin-converting enzyme 2 expression with $<1 \%$ angiotensin-converting enzyme 2 positive cells. ${ }^{4}$ High expression of angiotensinconverting enzyme 2 and direct communication of gastrointestinal tract with external environment makes it more susceptible to infection by virus.

Though less common, patients affected with Covid-19 have presented with symptoms such as nausea and abdominal pain, diarrhoea and vomiting. Some patients presented with the complaints of diarrhoea and nausea and later developed fever and dyspnea. ${ }^{13}$ A man presented with a 4-day history of diarrhoea and low fever only, was later diagnosed with Covid$19^{41}$ shows the early involvement of gastrointestinal tract in disease.

Studies have shown that faecal specimens are as accurate as pharyngeal specimens for Covid-19 detection. ${ }^{42}$ While another study showed positive stool specimens in a Covid-19 asymptomatic boy even when respiratory tract specimens were negative by reverse transcription-polymerase chain (RT-PCR). 43

Specific angiotensin-converting enzyme 2 receptors are identified in cholangiocytes and hepatocytes while Kupffer cells and endothelial cells of liver were found to be angiotensin-converting enzyme 2 negative. ${ }^{4}$ Gammaglutamyltransferase (GGT) levels were found to be increased in some Covid-19 patients ${ }^{45}$ which represents that coronavirus may cause liver damage.

Virus can enter the gastrointestinal system directly or indirectly via systemic circulation. Angiotensin-converting enzyme 2 receptors are abundantly present in lower gastrointestinal tract specifically in ileum. angiotensin- converting enzyme 2 / Angiotensin 1 - 7 axis modulates the immune response by influencing the composition of gut microbiota, explains the pathophysiology of various diseases including inflammation of intestines and diarrhea. ${ }^{46}$ Angiotensin-converting enzyme 2 is the regulator of gut microbiota composition as well as innate immunity and inactivation of angiotensin-converting enzyme 2 results in severe colitis following intestinal inflammation. ${ }^{47}$ This is one of the possible explanations for gastrointestinal related manifestations in patients with Covid-19. Other explanations include cytokine storm which can cause intestinal inflammation. Inflammation of small bowel and colon and the alteration of the gut microbiome lead to an imbalance of the innate immune system of the gut and more systemic inflammation. 48

\section{PREGNANCY AND COVID-19}

Pregnant women and neonates are more susceptible to pathogens of respiratory tract than general population and hence they are more prone to development of pneumonia, which increases their chances of Covid-19 infection. ${ }^{49}$ Serious complications during pregnancy, maternal rapid deterioration, premature births and even maternal deaths were noticed in severe acute respiratory syndrome infected pregnant females. ${ }^{50}$ However pregnant females with Covid-19 are showing fewer complications. In a study by Zhu et al. on 10 babies born to Covid-19 positive females, perinatal infection is found to have adverse effects and may cause premature labor, thrombocytopenia, abnormal liver functions and even death. ${ }^{51}$ No maternal to fetal transmission was noted in 29 babies tested for Covid born from Covid positive mothers at China. ${ }^{52}$ Neither of severe acute respiratory syndrome and Middle East respiratory syndrome had shown this vertical transmission of disease. Because of more susceptibility of pregnant females and neonates to Covid-19 they should be considered as highrisk population and more focus should be provided in prevention and management of disease in them.

Covid-19 caused by Severe Acute Respiratory Syndrome Coronavirus-2 (SARS-CoV-2) is found to affect various organ systems. Though the underlying mechanisms are not very clear but the possible reasons for involvement of more than one organ systems include, firstly the wide distribution of angiotensin-converting enzyme 2 receptors in human body. Secondly, the hyperactive immune system and cytokine storm which may lead to tissue necrosis and inflammation of various organs. Thirdly, the hypercoagulability of blood may lead to thrombosis and hypoxia in various tissues. Various other mechanisms, secondary or independent of these mechanisms may also contribute to the pathophysiology of multiple organ dysfunctions.

Patients with hypertension, cardiovascular diseases and cerebrovascular diseases are at higher risk for Covid-19 infection. ${ }^{53}$ Aged individuals, pregnant females and neonates are also among the high-risk groups.

Though exact mechanism of cytokine storm, maternal to fetal transmission, hypercoagulability etc. are still unknown. More studies on the mechanisms by which SARS-CoV-2 affects various organs and its modes of transmission can help us to establish better preventive measures and management 
strategies. We recommend individuals and clinicians to consider the risk of Covid-19 infection, even if common pulmonary symptoms are absent. Also more studies emphasizing involvement of extra pulmonary systems and related possible symptoms can help both the patients and clinicians in early diagnosis of disease.

Financial or other competing interests: None.

Disclosure forms provided by the authors are available with the full text of this article at jemds.com.

\section{REFERENCES}

[1] WHO. WHO Coronavirus Disease (Covid-19) Dashboard. https://covid19.who.int/?gclid

[2] Zhang $S$, Diao $M, Y u ~ W$, et al. Estimation of the reproductive number of novel coronavirus (COVID - 19) and the probable outbreak size on the Diamond Princess cruise ship: a data-driven analysis. Int J Infect Dis 2020;93:201-4

[3] Walls AC, Park YJ, Tortorici MA, et al. Structure, function and antigenicity of the sars-coV-2 spike glycoprotein. Cell 2020;181(2):281-92.

[4] Zou X, Chen K, Zou J, et al. Single-cell RNA-seq data analysis on the receptor angiotensin-converting enzyme 2 expression reveals the potential risk of different human organs vulnerable to 2019-nCoV infection. Front Med 2020;14(2):185-92.

[5] Valdés G, Neves LAA, Anton L, et al. Distribution of angiotensin-(1-7) and angiotensin-converting enzyme 2 in human placentas of normal and pathological pregnancies. Placenta 2006;27(2-3):200-7.

[6] Ding Y, He L, Zhang Q, et al. Organ distribution of severe acute respiratory syndrome (SARS) associated coronavirus (SARS-CoV) in severe acute respiratory syndrome patients: implications for pathogenesis and virus transmission pathways. J Pathol 2004;203(2):62230.

[7] Qin C, Zhou L, Hu Z, et al. Dysregulation of immune response in patients with coronavirus 2019 (Covid-19) in Wuhan, China. Clin Infect Dis 2020;71(15):762-8.

[8] Du Y, Tu L, Zhu P, et al. Clinical features of 85 fatal cases of COVID - 19 from Wuhan. A retrospective observational study. Am J Respir Crit Care Med 2020;201(11):1372-9.

[9] Tisoncik JR, Korth MJ, Simmons CP, et al. Into the eye of the cytokine storm. Microbiol Mol Biol Rev 2012;76(1):16-32.

[10] Halyabar O, Chang MH, Schoettler ML, et al. Calm in the midst of cytokine storm: a collaborative approach to the diagnosis and treatment of hemophagocytic lymphohistiocytosis and macrophage activation syndrome. Pediatr Rheumatol Online J 2019;17(1):7.

[11] Chen L, Liu HG, Liu W, et al. Analysis of clinical features of 29 patients with 2019 novel coronavirus pneumonia. Zhonghua Jie He He Hu Xi Za Zhi 2020;43(0):E005.

[12] Ruan Q, Yang K, Wang W, et al. Clinical predictors of mortality due to COVID - 19 based on an analysis of data of 150 patients from Wuhan, China. Intensive Care Med 2020;46(5):846-8.

[13] Wang D, Hu B, Hu C, et al. Clinical characteristics of 138 hospitalized patients with 2019 novel coronavirus- infected pneumonia in Wuhan, China. JAMA 2020;323(11):1061-9.

[14] Peiris J, Lai S, Poon L, et al. Coronavirus as a possible cause of severe acute respiratory syndrome. Lancet 2003;361(9366):1319-25.

[15] Zhou F, Yu T, Du R, et al. Clinical course and risk factors for mortality of adult inpatients with Covid-19 in Wuhan, China: a retrospective cohort study. Lancet 2020;395(10229):1054-62.

[16] Fujishima S. Pathophysiology and biomarkers of acute respiratory distress syndrome. Journal of Intensive Care 2014;2(1):32.

[17] Martin TR. Interactions between mechanical and biological processes in acute lung injury. Proc Am Thorac Soc 2008;5(3):291-6.

[18] Chan MC, Hsu JY, Liu HH, et al. Effects of prone position on inflammatory markers in patients with ARDS due to community-acquired pneumonia. J Formos Med Assoc 2007;106(9):708-16.

[19] Badawi A, Ryoo SG. Prevalence of comorbidities in the middle East respiratory syndrome coronavirus (MERSCoV): a systematic review and meta-analysis. Int J Infect Dis 2016;49:129-33.

[20] Yang J, Zheng Y, Gou X, et al. Prevalence of comorbidities and its effects in patients infected with SARS-CoV-2: a systematic review and meta-analysis. Int J Infect Dis 2020;94:91-5.

[21] Inciardi RM, Lupi L, Zaccone G, et al. Cardiac involvement in a patient with coronavirus disease 2019 (COVID - 19). JAMA Cardiol 2020;5(7):819-24.

[22] Zheng YY, Ma YT, Zhang JY, et al. Covid-19 and the cardiovascular system. Nat Rev Cardiol 2020;17(5):25960.

[23] Lazzerini PE, Capecchi PL, El-Sherif N, et al. Emerging arrhythmic risk of autoimmune and inflammatory cardiac channelopathies. J Am Heart Assoc 2018;7(22):e010595.

[24] Lazzerini PE, Laghi-Pasini F, Boutjdir $M$, et al. Cardioimmunology of arrhythmias: the role of autoimmune and inflammatory cardiac channelopathies. Nat Rev Immunol 2019;19(1):63-4.

[25] Terpos E, Ntanasis-Stathopoulos I, Elalamy I, et al. Hematological findings and complications of Covid-19. Am J Hematol 2020;95(7):834-47.

[26] Wichmann D, Sperhake JP, Lütgehetmann M, et al. Autopsy findings and venous thromboembolism in patients with Covid-19. Ann Intern Med 2020;173(4):268-77.

[27] Helms J, Tacquard C, Severac F, et al. High risk of thrombosis in patients with severe SARS-CoV-2 infection: a multicenter prospective cohort study. Intensive Care Med 2020;46(6):1089-98.

[28] Du F, Liu B, Zhang S. Covid-19: the role of excessive cytokine release and potential angiotensin-converting enzyme 2 down - regulation in promoting hypercoagulable state associated with severe illness. J Thromb Thrombolysis 2020:1-17.

[29] Gu J, Gong E, Zhang B, et al. Multiple organ infection and the pathogenesis of SARS. J Exp Med 2005;202(3):415-24.

[30] Mao L, Jin H, Wang M, et al. Neurologic manifestations of hospitalized patients with coronavirus disease 2019 in Wuhan, China. JAMA Neurol 2020;77(6):683-90. 
[31] Camdessanche JP, Morel J, Pozzetto B, et al. COVID - 19 may induce Guillain-Barré syndrome. Rev Neurol (Paris) 2020;176(6):516-8.

[32] Filatov A, Sharma P, Hindi F, et al. Neurological complications of coronavirus disease (Covid-19): encephalopathy. Cureus 2020;12(3):e7352.

[33] Moriguchi T, Harii N, Goto J, et al. A first case of meningitis/encephalitis associated with SARS coronavirus-2. Int J Infect Dis 2020;94:55-8.

[34] Perlman S, Jacobsen G, Afifi A. Spread of a neurotropic murine coronavirus into the CNS via the trigeminal and olfactory nerves. Virology 1989;170(2):556-60.

[35] Li YC, Bai WZ, Hirano N, et al. Neurotropic virus tracing suggests a membranous-coating-mediated mechanism for transsynaptic communication. J Comp Neurol 2013;521(1):203-12.

[36] Cheng Y, Luo R, Wang K, et al. Kidney disease is associated with in-hospital death of patients with Covid-19. Kidney Int 2020;97(5):829-38.

[37] Chu KH, Tsang WK, Tang CS, et al. Acute renal impairment in coronavirus-associated severe acute respiratory syndrome. Kidney Int 2005;67(2):698-705.

[38] Lely AT, Hamming I, van Goor H, et al. Renal angiotensinconverting enzyme 2 expression in human kidney disease. J Pathol 2004;204(5):587-93.

[39] Gembardt F, Sterner-Kock A, Imboden H, et al. Organspecific distribution of angiotensin-converting enzyme 2 mRNA and correlating peptidase activity in rodents. Peptides 2005;26(7):1270-77.

[40] Su H, Yang M, Wan C, et al. Renal histopathological analysis of 26 postmortem findings of patients with Covid-19 in China. Kidney Int 2020;98(1):219-27.

[41] Song Y, Liu P, Shi XL, et al. SARS-CoV-2 induced diarrhoea as onset symptom in patient with Covid-19. Gut 2020;69(6):1143-4.

[42] Zhang J, Wang S, Xue Y. Fecal specimen diagnosis 2019 novel coronavirus-infected pneumonia. J Med Virol 2020;92(6):680-2.
[43] Tang A, Tong Z, Wang H, et al. Detection of novel coronavirus by RT-PCR in stool specimen from asymptomatic child, China. Emerg Infect Dis J 2020;26(6):1337-9.

[44] Chai X, Hu L, Zhang Y, et al. Specific angiotensinconverting enzyme 2 expression in cholangiocytes may cause liver damage after 2019- nCoV infection. BioRxiv 2020.

[45] Zhang C, Shi L, Wang FS. Liver injury in Covid-19: management and challenges. Lancet Gastroenterol Hepatol 2020;5(5):428-30.

[46] Andrade JMO, de Farias Lelis D, Mafra V, et al. The angiotensin converting enzyme 2 (angiotensinconverting enzyme 2), gut microbiota and cardiovascular health. Protein Pept Lett 2017;24(9):827-32.

[47] Hashimoto T, Perlot T, Rehman A, et al. angiotensinconverting enzyme 2 links amino acid malnutrition to microbial ecology and intestinal inflammation. Nature 2012;487(7408):477-81.

[48] Mönkemüller K, Fry LC, Rickes S. Covid-19, Coronavirus, SARS-CoV-2 and the small bowel. Rev Esp Enferm Dig 2020;112(5):383-8.

[49] Qiao J. What are the risks of Covid-19 infection in pregnant women? Lancet 2020;395(10226):760-2.

[50] Wong SF, Chow KM, de Swiet M. Severe acute respiratory syndrome and pregnancy. BJOG 2003;110(7):641-2.

[51] Zhu H, Wang L, Fang C, et al. Clinical analysis of 10 neonates born to mothers with 2019-nCoV pneumonia. Transl Pediatr 2020;9(1):51-60.

[52] Schwartz DA. An analysis of 38 pregnant women with Covid-19, their newborn infants, and maternal-fetal transmission of SARS-cov-2: maternal coronavirus infections and pregnancy outcomes. Arch Pathol Lab Med 2020;144(7):799-805.

[53] Fang L, Karakiulakis G, Roth M. Are patients with hypertension and diabetes mellitus at increased risk for Covid-19 infection? Lancet Respir Med 2020;8(4):e21. 